\title{
AVALIAÇÃO ECONÔMICA DAS ATIVIDADES DE USO INDIRETO EM UMA RESERVA PARTICULAR DO PATRIMÔNIO NATURAL
}

\author{
Keila Lima Sanches ${ }^{1}$, Álvaro Nogueira de Souza², Antônio Donizette de Oliveira ${ }^{3}$, Ana Paula Silva Camelo
}

(recebido: 18 de janeiro de 2010; aceito: 25 de fevereiro de 2011)

\begin{abstract}
RESUMO: Objetivou-se, com este estudo, avaliar a viabilidade econômica das atividades de uso indireto desenvolvidas em uma reserva particular do patrimônio natural - RPPN. As atividades desenvolvidas na RPPN são a Trilha Ecológica e o Turismo de Aventura. Obtiveram-se informações relacionadas ao número anual de pessoas que visitam a reserva, preços pagos para participar das atividades, custo da terra, custos de manutenção e custos com mão de obra. Os critérios econômicos utilizados foram o Valor Presente Líquido (VPL) e o Benefício Periódico Equivalente (BPE). No período 1996-2008, o número de visitantes aumentou 6\% ao ano, e a média anual de visitas à RPPN foi de 8.889 pessoas. Concluiu-se que as atividades de uso indireto da RPPN são economicamente viáveis e podem concorrer com outras atividades de uso direto do solo, como o plantio de eucalipto.
\end{abstract}

Palavras-chave: Unidade de conservação, turismo ecológico, trilha ecológica, turismo de aventura.

\section{ECONOMIC EVALUATION OF INDIRECT USE ACTIVITIES IN A PRIVATE NATURAL HERITAGE RESERVE}

\begin{abstract}
This study aimed to evaluate the economic viability of indirect use activities as developed in a private natural heritage reserve (RPPN). Activities developed in the RPPN include Adventure Tourism and an Ecological Trail. Data were obtained relating to annual number of people visiting the reserve, prices paid to participate in activities, cost of land, maintenance costs and labor costs. Economic criteria used include Net Present Value (VPL) and Equivalent Periodic Benefit (BPE). In the 19962008 period the number of visitors increased by $6 \%$ a year, and the average annual number of visitors to the RPPN was 8,889. It was concluded that indirect use activities in the RPPN are economically viable and can coexist with other direct soil use activities such as eucalyptus cultivation.
\end{abstract}

Key words: Conservation unit, ecological tourism, ecological trail, adventure tourism.

\section{INTRODUÇÃO}

O turismo, de modo geral, é uma das atividades econônicas mais importantes do mundo. A Organização Mundial do Turismo (OMT) estimou que 656 milhões de turistas internacionais geraram, em 1999, uma receita mundial de cerca de US\$ 456 bilhões. Nesse contexto, estima-se ainda que o número de turistas crescerá, nas próximas duas décadas, 4,2\% ao ano e a receita gerada por eles, cerca de 6,7\% ao ano (EMBRATUR, 2008).

O ecoturismo é um segmento da atividade turística que utiliza, de forma sustentável o patrimônio natural e cultural; incentiva sua conservação e busca a formação de uma consciência ambientalista. No Brasil, esse fenômeno turístico cresce muito rápido, sendo visto como uma alternativa para a conservação e o desenvolvimento sustentável, especialmente em áreas onde a população local é solicitada a abrir mão do uso tradicional e predatório dos recursos naturais em favor de outros tipos de uso. A principal causa do crescimento dessa atividade é o desejo dos turistas de interagir com a natureza, tendo como principal espaço de atuação áreas naturais conservadas. Esta condição realça o potencial do ecoturismo no país, com boa perspectiva econômica e político-ambiental, já que estas atividades impulsionam o aumento de áreas conservadas (LEE; MIELDE, 2007; MARTINS, 2003; WALLACE; PIERCE, 1996).

Os estudos de Lima e Pozzobon (2005) apontaram que a falta de opções econômicas para a sobrevivência imediata das populações resulta em pobreza e degradação ambiental, uma vez que, para os autores, as mesmas estão intimamente relacionadas.

\footnotetext{
${ }^{1}$ Engenheira Florestal, Doutoranda em Ciências Florestais - Departamento de Engenharia Florestal - Universidade de Brasília/UnB - Cx. P. 4357 70910-900 - keila.sanches@gmail.com

${ }^{2}$ Engenheiro Florestal, Professor Dr. em Engenharia Florestal - Departamento de Engenharia Florestal - Universidade de Brasília/UnB - Cx. P. 4357 70910-900-ansouza@unb.br

${ }^{3}$ Engenheiro Florestal, Professor Dr. em Ciência Florestal - Departamento de Ciências Florestais - Universidade Federal de Lavras - Cx. P. 3037 37200-000 - Lavras, MG - donizete@dcf.ufla.br

${ }^{4}$ Engenheira Florestal, Mestranda em Ciências Florestais - Departamento de Engenharia Florestal - Universidade de Brasília/UnB - Cx. P. 4357 70910-900 - paulaflorestal@gmail.com
}

Cerne, Lavras, v. 17, n. 2, p. 223-229, abr./jun. 2011 
A criação de Unidades de Conservação - UCs, em específico as Reservas Particulares do Patrimônio Natural - RPPN, tem sido intensificada com o crescimento do setor de turismo ecológico. De acordo com Mesquita e Leopoldino (2002) as RPPNs conquistaram o status de UCs com a aprovação da Lei n 9.985/2000, que instituiu o Sistema Nacional de Unidades de Conservação - SNUC. Passaram a ser consideradas unidades de conservação, integrantes do grupo de uso sustentável, fazendo do Brasil o único país da América Latina a incluir as reservas privadas no seu sistema oficial de áreas protegidas. Segundo a Confederação Nacional de RPPN - CNRPPN (2009) existem mais de 750 unidades de conservação particulares cadastradas no Brasil cuja a área total protegida é superior a 580 mil hectares. Desse total, o Bioma Cerrado possui cerca de 134 RPPNs, totalizando mais de 100 mil hectares.

As RPPNs são importantes para a conservação porque contribuem para uma rápida ampliação das áreas protegidas no país; atuam como zonas-tampão no entorno de parques e reservas, constituindo-se em corredores ecológicos; são facilmente regulamentadas; possibilitam a participação da iniciativa privada no esforço nacional de conservação e contribuem para a compensação da biodiversidade dos biomas brasileiros. Além disso, de acordo com o Art. $3^{\circ}$ do Decreto Federal 1.922/96, os proprietários de RPPNs dispõem de uma série de benefícios como: isenção do Imposto sobre a Propriedade Territorial Rural - ITR; prioridades na análise de concessão de recursos ao Fundo Nacional do Meio Ambiente - FNMA quando da análise de projetos necessários à implantação e gestão das RPPNs reconhecidas ou certificadas pelo IBAMA e preferência na análise do pedido de concessão de crédito agrícola, pelas instituições oficiais de crédito.

Pelo exposto, fica evidente a importância das RPPNs para a conservação dos recursos naturais e de seu potencial para gerar renda, por meio da implementação de atividades relacionadas ao turismo ecológico. Assim, realizou-se este estudo com o objetivo de avaliar a viabilidade econômica das atividades de uso indireto desenvolvidas em uma RPPN, segundo a definição do Sistema Nacional de Unidades de Conservação - SNUC (2000).

\section{MATERIAL E MÉTODOS}

\section{1 Área de estudo}

A área de estudo é uma RPPN de 17 hectares constituída por uma mata ciliar primária, com grande diversidade de fauna e flora. Ela se situa em uma propriedade rural denominada Fazenda Vagafogo, localizada no município de Pirenópolis, estado de Goiás, nas coordenadas de 1551'09” de latitude sul e 4857'33” de longitude oeste de Greenwich, conforme pode ser vista nas Figuras 1 e 2.

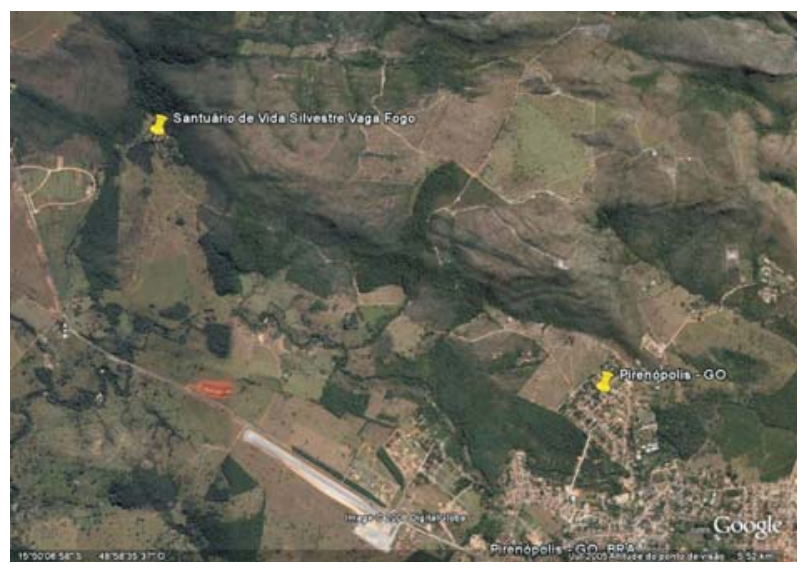

Figura 1 - Localização da Fazenda Vagafogo, a 5 Km do Município de Pirenópolis.

Figure 1 - Location of Fazenda Vagafogo, $5 \mathrm{Km}$ away from the municipality of Pirenópolis.

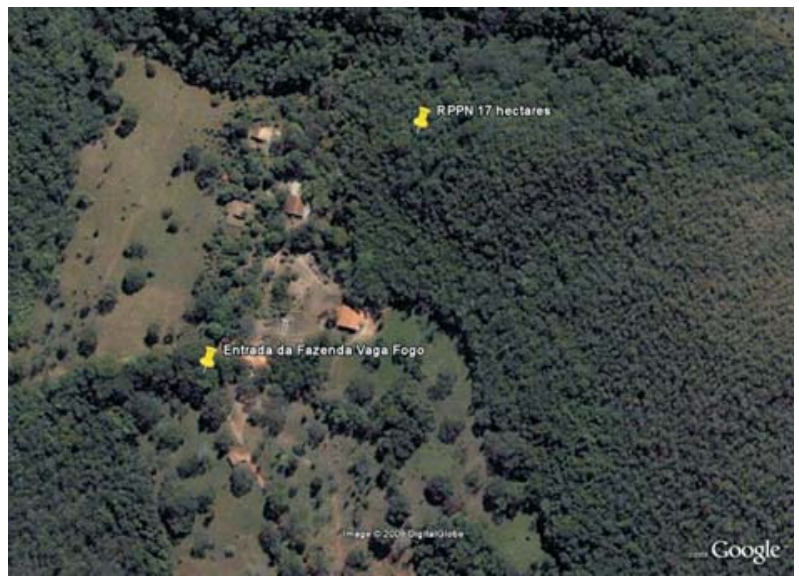

Figura 2 - Visualização parcial da Fazenda Vagafogo e de sua RPPN.

Figure 2 - Partial view of Fazenda Vagafogo and its RPPN.

A altitude média é de 770 metros e a área está inserida na mesorregião do Leste Goiano e na microrregião do entorno do Distrito Federal. O clima da região é quente

Cerne, Lavras, v. 17, n. 2, p. 223-229, abr./jun. 2011 
e enquadra-se no tipo Aw, variando de úmido a semi-árido, com até cinco meses de seca. A temperatura média anual no município de Pirenópolis é de $22^{\circ} \mathrm{C}$, variando de $16^{\circ} \mathrm{C}$ a $34^{\circ} \mathrm{C}$, e a precipitação média anual é de $1800 \mathrm{~mm}$, com um período de estiagem que vai de maio a setembro. A região é composta por diferentes ecossistemas do Cerrado: cerrado sensu stricto, cerradão, florestas ciliares, matas de galeria e florestas semideciduais (INSTITUTO NACIONAL DE METEOROLOGIA - INMET, 2008).

\subsection{Análise econômica}

As atividades desenvolvidas na RPPN em estudo são a trilha ecológica e o turismo de aventura (arvorismo, rapel e salto do primata). Os dados referentes ao número de visitantes, valor médio cobrado por visitante e custos operacionais para estas atividades foram obtidos a partir do acesso às informações de um banco de dados fornecido pela Fazenda Vagafogo. Todos os dados são registrados diariamente pelo empreendimento.

Como custo anual da terra, consideraram-se os juros sobre o seu valor. O preço da terra foi obtido pela base de dados da Fundação Getúlio Vargas, sendo de R\$ 2.600,00 por hectare para cerrado mata nativa, no Estado de Goiás, referente ao ano de 2008.

Para a análise da viabilidade econômica das atividades de turismo de aventura e trilha ecológica, foram utilizados os métodos do Valor Presente Líquido (VPL) e Benefício Periódico Equivalente (BPE) e taxa de juros de $8 \%$ ao ano, tomando-se como base as taxas utilizadas para análise de investimento de projetos florestais (REZENDE; OLIVEIRA, 2008).

$$
\begin{aligned}
& V P L=\sum_{j=0}^{n} R j(1+i)^{-j}-\sum_{j=0}^{n} C j(1+i)^{-j} \\
& B P E=\frac{V P L \cdot i(1+i)^{n}}{(1+i)^{n}-1}
\end{aligned}
$$

em que:

$C j$ = custos no final do período de tempo considerado;

$R j$ = receita no final do período de tempo considerado;

$i=$ taxa de juros;

$j$ = período em que as receitas ou os custos ocorrem;

$n=$ duração do projeto em anos.

Para verificar a quantidade mínima de usuários que viabilizam economicamente os pacotes de atividades desenvolvidos na RPPN, considerando um horizonte de planejamento de 10 anos, utilizou-se a seguinte equação:

$$
Q=\frac{C_{1} \frac{\left[(1+i)^{j}-1\right]}{i}+C_{2} \frac{\left[(1+i)^{j}-1\right]}{i}+\ldots+C_{n} \frac{\left[(1+i)^{j}-1\right]}{i}}{P \frac{\left[(1+i)^{j}-1\right]}{i}}
$$

em que:

$Q=$ Quantidade de usuários do pacote de atividades;

$P=$ Preço unitário cobrado pelo pacote de atividades;

$C_{1} ; C_{2} ; C_{n}=$ Custos relacionados às atividades;

$i=$ taxa de juros;

$j$ = período em que as receitas ou os custos ocorrem.

\section{RESULTADOS E DISCUSSÃO}

A Tabela 1 mostra o número de pessoas que visitaram a RPPN no período 1996-2008, e a Figura 3 expressa esses valores de forma gráfica. Verifica-se que até 2004 houve crescimento do número de visitantes, mas, nos anos seguintes, houve uma pequena queda. O número de visitantes aumentou em média $6 \%$ ao ano, e a média anual de visitas à RPPN foi de 8.889 pessoas.

Tabela 1 - Número anual de visitantes à RPPN no período 1996-2008.

Table 1 - Annual number of visitors to the RPPN in the 19962008 period.

\begin{tabular}{lc}
\hline Ano & Numero de Visitantes \\
\hline 1996 & 5.200 \\
1997 & 5.787 \\
1998 & 6.436 \\
1999 & 7.466 \\
2000 & 8.440 \\
2001 & 10.185 \\
2002 & 10.483 \\
2003 & 10.136 \\
2004 & 11.646 \\
2005 & 9.946 \\
2006 & 9.481 \\
2007 & 10.485 \\
2008 & 9.872 \\
\hline Total & 115.563 \\
\hline
\end{tabular}




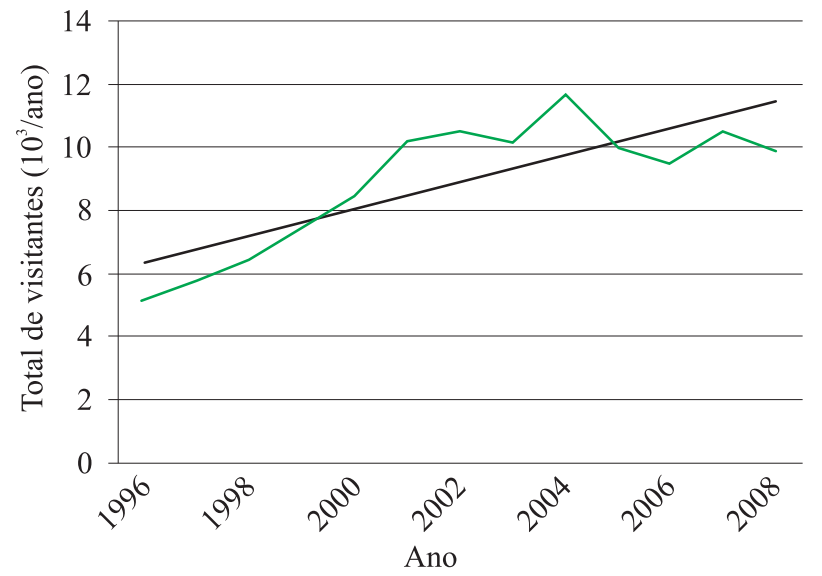

Figura 3 - Número de visitantes à RPPN no período 1996-2008.

Figure 3 - Number of visitors to the RPPN in the 1996-2008 period.

Na Tabela 2, constata-se que a maior parte das pessoas que visitaram a RPPN no período 2005-2008 pagou para participar de alguma atividade. A Tabela 3 indica os preços cobrados para usufruir das diversas atividades disponíveis na reserva. Para corrigir as variações no preço das atividades nos anos anteriores a 2008, utilizou-se um índice deflator de preço, o Índice Geral de Preços-Disponibilidade Interna (IGP-DI), tomando como ano-base 2008.

Tabela 2 - Dados anuais do número de visitantes à RPPN.

Table 2 - Annual data on number of visitors to the RPPN.

\begin{tabular}{cccc}
\hline \multirow{2}{*}{ Ano } & \multirow{2}{*}{$\begin{array}{c}\text { Visitantes } \\
\text { pagantes e } \\
\text { não-pagantes }\end{array}$} & \multicolumn{2}{c}{ Visitantes pagantes } \\
\cline { 3 - 4 } & $\begin{array}{c}\text { Trilha } \\
\text { Ecológica }\end{array}$ & $\begin{array}{c}\text { Turismo de } \\
\text { Aventura }\end{array}$ \\
\hline 2005 & 9.946 & 6266 & 2600 \\
2006 & 9.481 & 5404 & 2714 \\
2007 & 10.485 & 7025 & 1681 \\
2008 & 9.872 & 5923 & 1883 \\
\hline
\end{tabular}

Tabela 3 - Preço das atividades (R\$) praticadas na RPPN, em 2008.

Table 3 - Price of activities ( $R \$$ ) practiced in the RPPN in 2008.

\begin{tabular}{|c|c|c|c|c|c|}
\hline \multicolumn{4}{|c|}{ Preço individual } & \multirow{2}{*}{$\begin{array}{c}\text { Preço do } \\
\text { Pacote } \\
\text { A1 }\end{array}$} & \multirow{2}{*}{$\begin{array}{c}\text { Preço do } \\
\text { Pacote } \\
\text { A2 }\end{array}$} \\
\hline Arvorismo & Rapel & $\begin{array}{l}\text { Salto do } \\
\text { primata }\end{array}$ & $\begin{array}{c}\text { Trilha } \\
\text { ecológica }\end{array}$ & & \\
\hline 40,00 & 40,00 & 20,00 & 10,00 & 70,00 & 80,00 \\
\hline
\end{tabular}

Como citado anteriormente, a propriedade oferece aos visitantes as atividades recreativas: trilha ecológica, turismo de aventura (arvorismo, rapel e salto do primata).

A trilha possui cerca de 1.530 metros e é percorrida sob as copas de árvores da mata ciliar primária que margeia o rio Vagafogo, podendo o visitante observar o movimento da fauna dentro da mata, além de usufruir da beleza natural das espécies vegetais típicas da região. Para o rapel, é utilizada uma plataforma instalada à $20 \mathrm{~m}$ de altura no alto de um Jatobá. A aventura inicia-se na base desse jatobá. Para o arvorismo utiliza-se um conjunto de passarelas, feitas basicamente de cabo de aço e madeira, cada passarela possui um nível de dificuldade diferente, em razão de sua construção, disposição das estruturas e dos movimentos necessários. Para o salto do primata, o praticante sobe em uma árvore e salta para pegar um trapézio, com uma projeção visual de mais de $15 \mathrm{~m}$ de altura.

Podem-se observar, na Tabela 2, que a trilha ecológica mostra uma demanda superior as atividades de turismo de aventura, em média 2,93 visitantes para trilha ecológica para cada visitante que usufrui do turismo de aventura. Uma das explicações para este fato é de que o turismo de aventura ainda é uma atividade insipiente. Outra explicação para isso pode ser pautada na classificação etária do público que visita o empreendimento, que em sua maioria são estudantes (até 12 anos) e família com crianças também dessa faixa etária. Sabendo-se que as atividades de turismo de aventura são mais atraentes para jovens (de 15 a 25 anos).

O visitante pode participar de uma ou mais atividades, mas se quiser optar por participar de todas as atividades do turismo de aventura haverá um desconto de $30 \%$ no valor a ser pago, ou seja, ele pagará a quantia de $\mathrm{R} \$ 70,00$. Esse conjunto de atividades relacionadas ao turismo de aventura foi denominado de "pacote A1". Se, além das atividades de turismo de aventura, o visitante quiser também caminhar na trilha ecológica, pagará o total de $\mathrm{R} \$ 80,00$. A esse conjunto de atividades denominou-se "pacote A2".

Na Tabela 4, estão os dados de receitas e custos relacionados às atividades desenvolvidas na RPPN. A Fazenda Vagafogo, onde foi criada a RPPN, mantém uma parceria com a empresa Drena Ecoturismo e Aventura, que é a responsável pela implementação das atividades de turismo de aventura. $\mathrm{O}$ acordo com esta empresa estipula que $30 \%$ da receita bruta do turismo de aventura deve ser utilizado para o pagamento por seus serviços.

Cerne, Lavras, v. 17, n. 2, p. 223-229, abr./jun. 2011 
Tabela 4 - Receita bruta e custos referentes às atividades do turismo de aventura no período 2005-2008.

Table 4-Gross revenue and costs referring to adventure tourism activities in the 2005-2008 period.

\begin{tabular}{ccccc}
\hline Ano & $\begin{array}{c}\text { Receita } \\
\text { bruta } \\
(\mathrm{R} \$)\end{array}$ & $\begin{array}{c}\text { Custos } \\
\text { drena } \\
(\mathrm{R} \$)\end{array}$ & $\begin{array}{c}\text { Custos de } \\
\text { manutenção } \\
(\mathrm{R} \$)\end{array}$ & $\begin{array}{c}\text { Custos de } \\
\text { mão de obra } \\
(\mathrm{R} \$)\end{array}$ \\
\hline 2005 & $78.038,00$ & $23.411,40$ & $15.607,60$ & $15.607,60$ \\
2006 & $79.809,80$ & $23.942,94$ & $15.961,96$ & $15.961,96$ \\
2007 & $56.373,48$ & $16.912,04$ & $11.274,70$ & $11.274,70$ \\
2008 & $64.651,12$ & $19.395,34$ & $12.930,22$ & $12.930,22$ \\
\hline
\end{tabular}

Os custos de manutenção e investimento na estrutura necessária às atividades desenvolvidas na RPPN correspondem a $20 \%$ do valor da receita bruta do turismo de aventura. Os custos com mão-de-obra, que incluem os serviços de guias turísticos, descontos concedidos, etc., também correspondem a $20 \%$ da receita bruta do turismo de aventura.

A Tabela 5 contém os resultados de VPL e BPE, totais e por hectare, para a RPPN. Os valores por hectare são úteis para a comparação com atividades econômicas convencionais para a mesma região. A lucratividade aumenta consideravelmente quando se considera a implementação conjunta das atividades de turismo de aventura e de trilha ecológica, saindo de um BPE de R\$ 1.067,69/ha.ano ${ }^{-1}$ para um BPE de R $\$ 4.012,70 /$ ha.ano $^{-1}$. A estratégia da Fazenda é estabelecer que a atividade de Turismo de Aventura cubra todos os custos de infraestrutura, pagamento de terceiros, etc. Desta forma, qualquer atividade que para ser implementada dependa dessa infra-estrutura, gerará uma receita líquida, já que seus custos serão cobertos pelo Turismo de Aventura, como ocorre no caso Turismo de Aventura + Trilha Ecológica.

Joaquim (2009) desenvolveu estudo em uma região de cerrado no estado de Goiás e encontrou que a implantação de floresta de eucalipto destinada à produção de carvão vegetal é viável economicamente, considerando uma taxa de juros mínima de atratividade de 8\% a.a. Os VPLs encontrados por hectare para os cortes realizados aos 6, 12 e 18 anos, foram de $\mathrm{R} \$ 3.544,45, \mathrm{R} \$ 6.360,00$ e $\mathrm{R} \$ 8.017,61$, respectivamente, e o $\mathrm{BPE} / \mathrm{ha} \cdot \mathrm{ano}^{-1}$ no primeiro corte foi de $\mathrm{R} \$ 766,72$, no segundo de $\mathrm{R} \$ 843,94$ e no terceiro de $\mathrm{R} \$ 855,50$. O autor afirma que a atividade de produção de carvão vegetal a partir de eucalipto na região do cerrado é economicamente viável, apresentando valores de VPL positivos, além desta atividade se mostrar competitiva com outras atividades convencionais na região do cerrado no estado do Goiás, como por exemplo, a atividade de produção de soja.

Comparando-se os resultados econômicos obtidos por Joaquim (2009) com os obtidos nesse estudo em relação ao uso indireto da RPPN, percebe-se que a exploração de turismo de aventura e trilha ecológica são atividades que podem competir, em termos de maior retorno econômico no uso da terra, com outras atividades convencionais da região do cerrado. Porém, há que se considerar a escala de produção e o tamanho das propriedades, bem como, a localização dos empreendimentos, que para a RPPN deste estudo é privilegiada devido a proximidade com grandes centros urbanos (Goiânia e Brasília) o que permite uma demanda considerável.

Vale ressaltar que as atividades florestais de produção apresentam peculiaridades que as diferenciam (CARVALHO et al., 2005). Podem-se destacar, para o mercado produtivo florestal, no geral: o longo prazo para o retorno econômico, os altos custos para a sua implantação, escolha de uma adequada taxa de juros a ser utilizada, a necessidade (demanda), a presença de externalidades, dentre outros. Diante destas restrições, é importante que surjam novas alternativas econômicas de uso da terra que possibilitem um bom desenvolvimento econômico local, conseguindo driblar tais limitações e que, ao mesmo tempo, contribuam para a conservação dos recursos naturais, assim como as atividades desenvolvidas pelo empreendimento ecoturístico deste estudo.

Tabela 5 - Resultados de VPL e BPE para as atividades desenvolvidas na RPPN, no período 2005-2008.

Table 5 - VPL and BPE results for activities developed in the RPPN, in the 2005-2008 period.

\begin{tabular}{lcccc}
\hline \multirow{2}{*}{ Atividades } & \multicolumn{2}{c}{ VPL (R\$) } & \multicolumn{2}{c}{ BPE (R\$) } \\
\cline { 2 - 5 } & Total & ha & Total & ha.ano $^{-1}$ \\
\hline Turismo de aventura & $60.117,59$ & $3.536,33$ & $18.150,75$ & $1.067,69$ \\
Turismo de aventura + Trilha & $225.939,92$ & $13.290,58$ & $68.215,96$ & $4.012,70$ \\
\hline
\end{tabular}

Cerne, Lavras, v. 17, n. 2, p. 223-229, abr./jun. 2011 
A Tabela 6 mostra os resultados da análise econômica para as atividades de Trilha Ecológica e Turismo de Aventura, em um horizonte de planejamento de 10 anos. Considerou-se a tomada de decisão no ano 2008 e taxas anuais de crescimento do número de usuários da trilha ecológica e do turismo de aventura iguais a 3,7\% e $1,26 \%$, respectivamente.

Tabela 6 - VPL e BPE para as atividades de turismo de aventura e trilha ecológica para o horizonte de planejamento de 10 anos.

Table 6 - VPL and BPE for adventure tourism and ecological trail activities over a 10-year planning horizon.

\begin{tabular}{lcccc}
\hline \multirow{2}{*}{ Atividades } & \multicolumn{2}{c}{ VPL (R\$) } & \multicolumn{2}{c}{ BPE (R\$) } \\
\cline { 2 - 5 } & Total & ha & Total & ha.ano-1 \\
\hline $\begin{array}{l}\text { Turismo de } \\
\text { Aventura }\end{array}$ & $69.360,93$ & $4.080,05$ & $10.336,82$ & 608,05 \\
$\begin{array}{l}\text { Turismo de } \\
\text { Aventura }+ \\
\text { Trilha }\end{array}$ & $434.392,54$ & $25.552,50$ & $64.737,30$ & $3.808,08$ \\
& & & & \\
\hline
\end{tabular}

Explorando-se apenas o Turismo de Aventura, obtém-se lucratividade bem menor que se for agregada também a opção de Trilha Ecológica. A associação das duas atividades eleva a lucratividade em cerca de seis vezes em relação à exploração apenas do Turismo de Aventura. Essa grande diferença ocorre devido ao fato de os administradores da Fazenda Vagafogo terem definido que os custos de infra-estrutura devem ser cobertos com a receita gerada pelo Turismo de Aventura. Dessa forma, qualquer atividade que, para ser implementada, dependa da infra-estrutura da Fazenda, gera uma receita líquida, já que seus custos serão cobertos pelo Turismo de Aventura.

Ao longo dos 10 anos analisados, o retorno das atividades de Turismo de Aventura e Trilha Ecológica supera o retorno encontrado para as atividades convencionais analisadas por Joaquim (2009) permitindo fortalecer a idéia de que a exploração econômica dessa RPPN é competitiva.

A Tabela 7 mostra a quantidade mínima de usuários que viabilizam economicamente os pacotes de atividades desenvolvidas na RPPN (ponto de equilíbrio). O pacote que reúne o turismo de aventura e a trilha ecológica necessita de uma quantidade menor de usuários que o pacote que oferece apenas o turismo de aventura, mostrando que é mais viável explorar as duas atividades em conjunto.

Cerne, Lavras, v. 17, n. 2, p. 223-229, abr./jun. 2011
Tabela 7 - Quantidade mínima de usuários para os pacotes de atividades.

Table 7 - Minimum number of users for the activity packages.

\begin{tabular}{ccc}
\hline \multirow{2}{*}{ Ano } & \multicolumn{2}{c}{ Número mínimo de usuários } \\
\cline { 2 - 3 } 2008 & A1 + A2 & A1 \\
\hline 2009 & 566 & 647 \\
2010 & 580 & 663 \\
2011 & 594 & 679 \\
2012 & 609 & 696 \\
2013 & 624 & 713 \\
2014 & 639 & 731 \\
2015 & 655 & 749 \\
2016 & 672 & 767 \\
2017 & 688 & 786 \\
\hline
\end{tabular}

Nota: A1 = Pacote Turismo de Aventura; A2 = Trilha Ecológica; A1 + A2 = Pacote Turismo de Aventura e Trilha.

Note: A1 = Adventure Tourism package; A2 = Ecologic Trail package; $A 1+A 2$ = Adventure Tourism and trail package.

\section{CONCLUSÕES}

A partir da análise da RPPN em estudo, foi possível mostrar que além de ser uma forma adequada de incentivar a conservação dos recursos naturais, as atividades de uso indireto desenvolvidas na propriedade são economicamente viáveis e podem concorrer com outras atividades de uso direto do solo, como o plantio de eucalipto. Além disso, verifica-se que há um potencial de crescimento para a rentabilidade originada pelas atividades de uso indireto em áreas preservadas, uma vez que o turismo ecológico se apresenta em crescimento.

\section{REFERÊNCIAS}

CARVALHO, R. M. M. A.; SOARES, T. S.; VALVERDE, S. R. Caracterização do setor florestal: uma abordagem comparativa com outros setores da Economia. Ciência Florestal, Santa Maria, v. 15, n. 1, p. 105-118, 2005.

CONFEDERAÇÃO NACIONAL DE RPPN. Disponível em: $<$ http://www.rppnbrasil.org.br/>. Acesso em: 10 jan. 2009.

EMBRATUR. Disponível em: <http://www.embratur.gov.br>. Acesso em: 26 maio 2008. 
JOAQUIM, M. S. Carvão vegetal: uma alternativa para os produtos rurais do sudoeste goiano. 2009. 97 p. Dissertação (Mestrado em Ciências Florestais) - Universidade de Brasília, Brasília, 2009.

INSTITUTO NACIONAL DE METEOROLOGIA. NORMAIS CLIMATOLÓGICAS. Disponível em: <http:// www.inmet.gov.br>. Acesso em: 15 jun. 2008.

LEE, C.; MIELDE, J. Valuation of ecotourism resources using a contingent valuation method: the case of the Korean DMZ. Ecological Economics, Cambridge, v. 63, p. 511-520, 2007.

LIMA, D.; POZZOBON, J. Amazônia socioambiental: sustentabilidade ecológica e diversidade social. Estudos Avançados, São Paulo, v. 19, n. 54, p. 45-76, 2005.

MARTINS, B. M. K. Desenvolvimento do Ecoturismo em RPPN's no Mato Grosso do Sul. 2003. 67 p.

Monografia (Graduação em Turismo) - Universidade para o
Desenvolvimento do Estado e da Região do Pantanal, Campo Grande, 2003.

MESQUITA, C. A. B.; LEOPOLDINO, F. S. Incentivando e apoiando criação, manejo e integração entre Reservas Particulares do Patrimônio Natural (RPPN). In: CONGRESSO BRASILEIRO DE UNIDADES DE CONSERVAÇÃO, 3., 2002. Anais... Rede Pró-Unidades de Conservação e Fundação O Boticário de Proteção à Natureza, 2002.

REZENDE, J. L. P.; OLIVEIRA, A. D. Análise econômica e social de projetos florestais. Viçosa, MG: UFV, 2008. 384 p.

SISTEMA NACIONAL DE UNIDADES DE

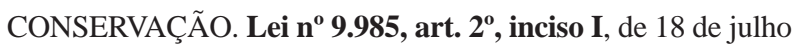
de 2000. Brasília, 2000. Disponível em: <http://www.planalto. gov.br/ccivil_03/LEIS/L9985.htm>. Acesso em: 10 jan. 2010.

WALLACE, G. N.; PIERCE, S. M. An evaluation of ecotourism in Amazonas, Brazil. Annals of Tourism Research, p. 843-873, 1996. 